\title{
Effect of high intensity interval training under hypoxic conditions in a normobaric environment on moderately trained university students' antioxidant status
}

\author{
Akgul M.S. ${ }^{1 \mathrm{ABCDE}}, \mathrm{Koz}$ M. ${ }^{2 \mathrm{ABCDE}}$ \\ 'School of Physical Education and Sports' Department of Coaching Education, Kastamonu University, Kastamonu, \\ Turkey \\ ${ }^{2}$ Faculty of Sport Sciences, Department of Coaching Education, Ankara University, Ankara, Turkey
}

Authors' Contribution: A - Study design; B - Data collection; C - Statistical analysis; D - Manuscript Preparation; E - Funds Collection.

\begin{abstract}
Purpose: $\quad$ The effects of high intensity interval exercises on antioxidant defence system are not clear. Since there is an evident lack of studies focused on oxidative stress in moderately trained males following high intensity interval training, we investigated oxidative stress markers (malondialdehyde [MDA], catalase [CAT], glutathione peroxidase [GPX], superoxide dismutase [SOD]) by completing a high intensity interval training program (HIITP) under hypoxic and normoxic conditions in a normobaric environment.

Material: $\quad$ The study was carried out on moderately trained university students who had regular exercising habits. The participants completed 8-week wingate based high intensity interval training under normoxic and hypoxic conditions $(2500 \mathrm{~m}$.) in the normobaric environment. They were instructed to maintain their normal dietary practices during the study not to take any antioxidant containing vitamin tablets.

Results: The interaction effect (timexgroup) for SOD $(p=0.230)$, CAT $(p=0.736)$, GPX $(p=0.517)$, and MDA ( $p=0.596)$, revealed no significant change in repeated response.

Conclusions: Although 8 weeks of high-intensity interval training significantly affected only SOD and GPX $(p<0.05)$, the normoxic and hypoxic conditions did not present any significant change between treatments.

Keywords: interval training, superoxide dismutase, catalase, glutathione peroxidase, malondialdehyde.
\end{abstract}

\section{Introduction}

In recent decades, intensive research in the field of oxidative damage indicates that exercise exacerbates the generation of reactive oxygen species (ROS) and reactive nitrogen species (RNS), some of which are free radicals $[1,2]$. A free-radical is any specie capable of existence with one or more unpaired electron [3]. ROS/RNS refer to oxygen or nitrogen containing free-radicals and their nonfree-radical derivatives [4]. ROS are generated by regular metabolic process in vivo and can initiate a cascade of free-radical formation and damage to macromolecules [5], Oxidative stress is an inevitable consequence of aerobic life, and there is growing evidence that the endogenous generation of ROS plays a major role in aging and many pathological conditions [6]. In resting state the body is equipped with both non-enzymatic and enzymatic antioxidant defence system to scavenge the potentially harmful effects of ROS [7, 8]. This system includes antioxidant enzymes such as glutathione peroxidase (GPX), catalase (CAT), and superoxide dismutase (SOD), and non-enzymatic molecules including vitamin $\mathrm{E}$, vitamin $\mathrm{C}$, vitamin A precursor, thiol-containing compounds e.g. glutathione (GSH). These antioxidant defence systems preserve homeostasis for normal cell functions at rest and under normal physiological conditions. However, during strenuous exercise, pathogenic processes and aging, ROS production may overwhelm antioxidant defence capacity causing cell and tissue damage $[9,10]$.

\footnotetext{
(c) Akgul M.S. , Koz M., 2019

doi:10.15561/20755279.2019.0501
}

Chronic aerobic exercise has emerged as a promising means of reducing oxidative stress. Mechanisms responsible for beneficial effects of chronic aerobic exercise are training induced regulation of SOD [11] and CAT [4] and reduced mitochondrial reactive oxygen species [12]. It has been proposed that chronic high intensity interval training (HIIT) may elicit greater health benefits than traditional chronic aerobic exercise, so HIIT has gained importance, since it is more effective on developing aerobic capacity. While it provides fast and effective adaptation, it also shortens exercise time. Nowadays, HIIT and its other forms are most effective training methods used to improve aerobic and anaerobic capacity, cardiovascular system and metabolic functions $[13,14]$. HIIT provides new and favorable contributions in respect to health and performance and positive adaptation for both sedentaries and athletes. When it is compared with traditional aerobic exercise, it has been drawing interest, since it uses time more economically and effectively, improves aerobic and anaerobic systems besides the metabolic functions and physical performance $[15,16]$. High intensity training can produce oxidative stress and antioxidant elements of organisms are affected with this challenge [17]. Few studies have investigated oxidative stress in response to both aerobic and anaerobic exercise bouts $[18,19]$, especially oxidative stress, which is experienced following sporting competitions [20].

Apart from abovementioned effects, intermittent hypoxia (IH) occurs in many pathophysiological conditions. The molecular mechanisms associated 
with $\mathrm{IH}$, however, have received little attention [21] The consequences of oxidative stress under hypoxic conditions, when physical effort is limited by the availability of oxygen to the working muscles, are of great interest to sport science [22-24]. Indeed, physical training under hypoxic conditions is frequently used to improve physical performance $[25,26]$ and training in hypoxic conditions has become an important element of preparing elite athletes $[27,28]$. The training is thought to be most effective when it is performed at an altitude of 2, 000 to 2, $500 \mathrm{~m}[29,30]$.

There have been studies concerning effects of HIIT and $\mathrm{IH}$ applications on antioxidant markers $[12,31]$. These studies investigated acute effects and were applied to elite athletes and thus there is a lack of studies investigating effects of this training method on antioxidant markers of active individuals for a longer time. Herein, we evaluated the antioxidant status after 8 week wingate style HIIT protocols in untrained healthy men. Plasma samples were collected for the measurement of CAT, SOD, GPX and MDA activities and total antioxidant status as a general marker of antioxidant defences.

\section{Material and Methods}

Subjects

In this study, 16 recreationally active university students volunteers, aged 20-29 (23.50 \pm 2.52$)$ were involved in this study. Only males were included to avoid any distortion in the hormonal response to physical exercise caused by sex differences. Anthropometric characteristics of the subjects are summarized in Table 1. The exclusion criteria for study were drugs and medicines intake as well as suffering from some illness and smoking habit. None of the subjects participated regularly in sport competitions and they did not engage in any form of vigorous exercise for 24 hours before the study was performed. They were instructed to maintain their normal dietary practices during the study and not to take any antioxidant containing vitamin tablets.

Table 1. Anthropometric characteristics of the subjects

\begin{tabular}{ll}
\hline Variables $(\mathbf{n = 1 6 )}$ & Mean \pm SD \\
\hline Age (years) & $23.50 \pm 2.52$ \\
Height $(\mathrm{cm})$ & $174.00 \pm 6.19$ \\
Body Weight $(\mathrm{kg})$ & $70.60 \pm 9.03$ \\
\hline
\end{tabular}

\section{Physiological measurements}

The body mass was measured using calibrated digital scales and height was measured using stadiometer. The age of athletes were accurately recorded as years. Blood samples were taken from the participants three times; the first one prior the training, the second one in 4th week and the third one after the training.

Exercise Protocol

16 participants were randomly assigned to normoxic or hypoxic groups and then they were completed 8-week high intensity interval training on normoxic and hypoxic conditions $(2500 \mathrm{~m})$ in the normobaric environment. The hypoxic conditions were provided with Hypoxica Submit II exercise package (Made in USA). All participants completed 8 weeks of wingate style cycling training, 3 days/week, consisting of incremental repeats 4 to 7 every two weeks $\times 30$ s all-out effort with 4 min rests.

\section{Measurement of oxidative status}

Erythrocyte MDA level were measured as previously described by Dahle et al. [32], SOD level was measured as previously described by Durak et al. [33], GPX level were measured as previously described by Paglia and Valantina [34], CAT level was measured as previously described by Aebi [35].

Statistical analysis

Results of all variables are expressed as mean and standard deviation. The Shapiro-Wilk Test of normality was used to determine if the data normally distributed. Baseline differences of antioxidant variables were calculated with Independent Sample T-Test between groups. Then the two way repeated measures ANOVA was used to compare differences in three measurement results using time and conditions factor (intraction effect: time $\times$ group). The post-hoc analysis was performed to specify pairwise differences. All analyses were set at $\mathrm{p}=0.05$ significance level.

\section{Results}

The baseline measurements of SOD, CAT, GPX, and MDA presented no significant differences in normoxic and hypoxic groups $(p>0.05)$. The interaction effect (time $\times$ group) for SOD $(p=0.230)$, CAT $(p=0.736)$, GPX $(\mathrm{p}=0.517)$, and MDA $(\mathrm{p}=0.596)$, revealed no significant change in repeated response (baseline, after 4 weeks and 8 weeks) (Table 2). Although 8 weeks of high-intensity interval training effected significantly only SOD and GPX $(p<0.05)$, the normoxic and hypoxic conditions did not present any change between treatments. The post-hoc analysis showed that the high-intensity interval training effect differed 4 weeks and 8 weeks for SOD $(p=0.037)$, baseline and 8 weeks for GPX $(p=0.014)$ responses. The rate of nonsignificant increase on SOD was $13.5 \%$ in the hypoxic group, $8.65 \%$ in the normoxic group after 8 weeks (Figure 1). This trend was not same for GPX response in hypoxic condition. The GPX decreased by $2.33 \%$. However, GPX was increased by $7.33 \%$ in the normoxic group (Figure 2). When we looked our study, we monitored that MDA levels increased both 4 th and 8 th week. $(\mathrm{p}<0.05)$

\section{Discussions}

To our knowledge, this is the first investigation to compare the differential effects of HIIT in hypoxia vs HIIT in normoxia on antioxidant status in a normobaric environment. There are studies stating that both methods changed oxidative stress markers [31, 36-38].

There are numerous reports that provide reasonable 
Table 2. The baseline measurements.

\begin{tabular}{|c|c|c|c|c|c|c|c|c|}
\hline \multirow[t]{2}{*}{ Indicators } & \multicolumn{3}{|c|}{ HIIT (Hypoxia) } & \multicolumn{5}{|c|}{ HIIT (Normoxia) } \\
\hline & Baseline & 4 Weeks & 8 Weeks & Baseline & 4 Weeks & 8 Weeks & $\begin{array}{l}\text { Timex } \\
\text { Treatment }\end{array}$ & d \\
\hline \multirow{2}{*}{$\mathrm{SOD}(\mathrm{U} / \mathrm{ml})$} & 1930.16 & 2046.95 & 2231.46 & 2449.82 & 2099.72 & 2681.93 & \multirow{2}{*}{$p=0.230$} & \multirow{2}{*}{0.20} \\
\hline & \pm 1183.74 & \pm 745.15 & \pm 714.93 & \pm 799.13 & \pm 1165.33 & \pm 859.85 & & \\
\hline \multirow{2}{*}{ CAT (KU/ml) } & 29691.75 & 27724.50 & 28685.25 & 32848.50 & 28273.50 & 31476.00 & \multirow{2}{*}{$p=0.736$} & \multirow{2}{*}{0.04} \\
\hline & \pm 5232.78 & \pm 6578.55 & \pm 8418.99 & \pm 8687.06 & \pm 4599.50 & \pm 7156.07 & & \\
\hline GPX (U/ml) & $6.47 \pm 1.67$ & $8.13 \pm 1.64$ & $6.27 \pm 2.14$ & $5.73 \pm 1.31$ & $6.81 \pm 1.69$ & $6.15 \pm 1.88$ & $p=0.517$ & 0.09 \\
\hline \multirow{2}{*}{ MDA (nmol/ml) } & 263.12 & 274.71 & 275.72 & 263.12 & 255.05 & 276.22 & \multirow{2}{*}{$p=0.596$} & \multirow{2}{*}{0.07} \\
\hline & \pm 21.41 & \pm 33.56 & \pm 24.18 & \pm 60.22 & \pm 34.47 & \pm 51.55 & & \\
\hline
\end{tabular}

Notes. SOD: superoxide dismutase; CAT: catalase; GPX: glutathione peroxidase; MDA: malondialdehyde. $n=16$
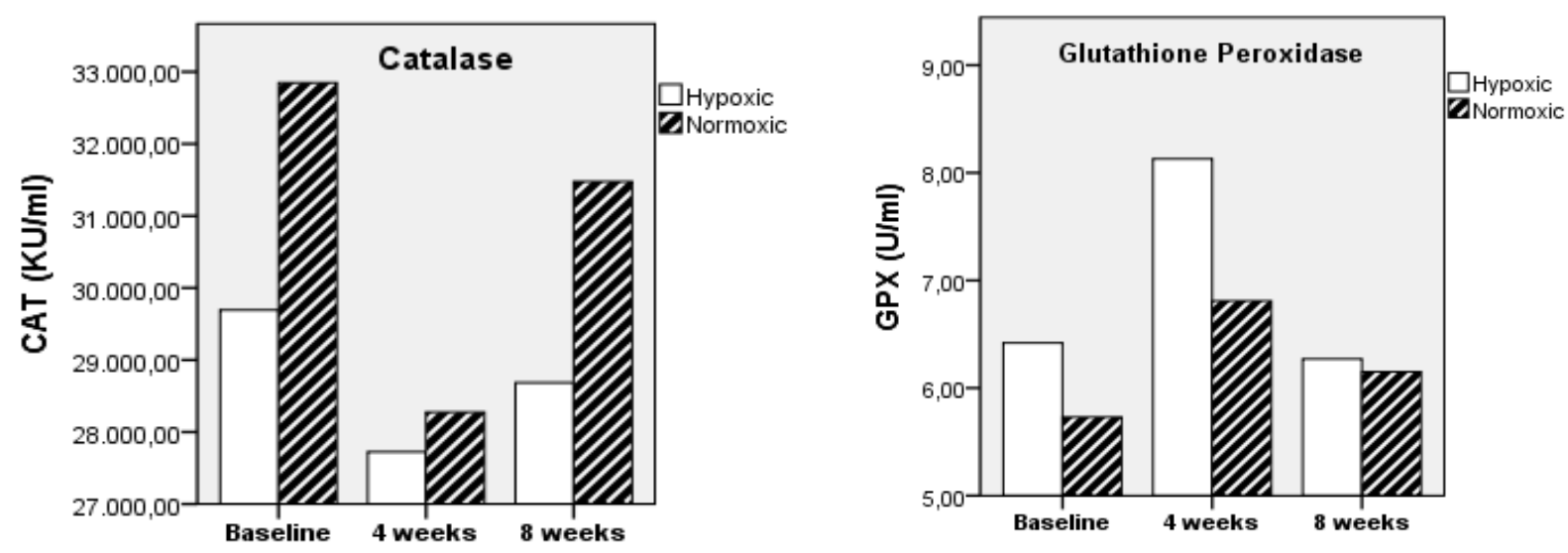

Figure 1. The oxidative stress status of CAT and GPX on moderately trained university students. Values are expressed as mean \pm S.D. CAT: catalese; GPX: glutathione peroxidase; $n=16$.
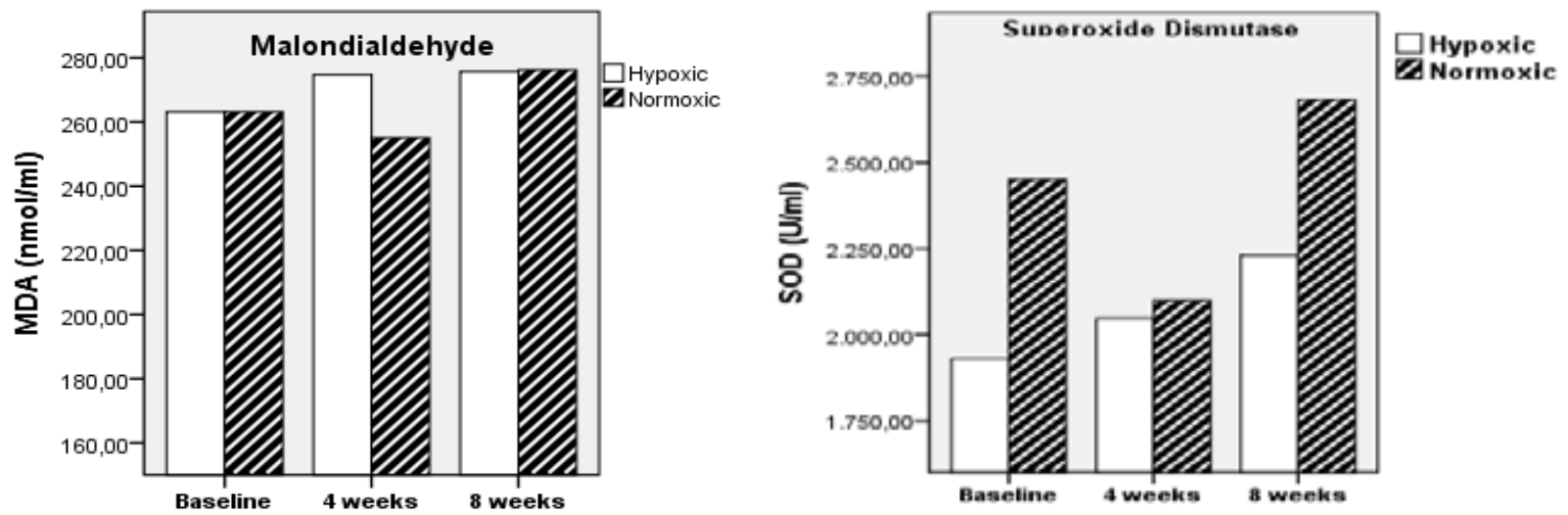

Figure 2. The oxidative stress of MDA and SOD on moderately trained university students Values are expressed as mean \pm S.D. MDA: melondialdehyde; SOD: superoxide dismutase; $n=16$.

support to the notion that exercise increases the production of ROS [39]. Little is known, regarding the extend of oxidative stress when comparing aerobic and anaerobic exercise modes [40]. On the occurrence of exercise, stress is not clear. However, the principal factor responsible for oxidative damage during exercise is the increase in oxygen consumption [41]. It appears that anaerobic types of exercise, which involves less oxygen circulation throughout the body than aerobic exercise is associated with an increased ROS generation level through other pathways [42, 43] suggesting that oxygen consumption per se is not the major cause of exercise induced oxidative damage [41].

Different types of exercises may have different effects on oxidative stress [34]. Which is defined as a situation in which an increased level of ROS generation overwhelms 
the antioxidant defence capacity, resulting in oxidative damage to lipits, proteins and DNA [41, 43].

A review of literature on changes in oxidative stress markers and physical parameters following the long duration HIIT training in hypoxia indicates a lack of information in this subject area. For this reason, we investigated the oxidative status of moderately trained males during 8 week by completing HIIT program.

It was reported that the activities of antioxidant enzymes including SOD, CAT and GSH peroxidase (GPX) increased with an acute bout of exercise in skeletal muscle, heart and liver [38].

SOD, CAT, GPX and MDA activities in response to exercise are variable. When we looked at our study, we observed that interaction effect (time $\times$ group) for SOD , CAT, GPX and MDA revealed no significant in repeated response $(\mathrm{p}<0.05)$ (baseline, after 4 weeks and 8 weeks). SOD and GPX activities effected significantly by HIIT. But the normoxic and hypoxic conditions were not present between treatments. HIIT effect were differ 4 weeks and 8 weeks for SOD ( $p=0.037)$, baseline and 8 weeks for GPX ( $p=0.014)$ responses. This trend was not same for GPX response in hypoxic condition. However, GPX was increased by non significantly in the normoxic group. MDA levels increased both 4th and 8 th week.

Currently, there is limited information on the effects of HIIT on the development of oxidative stress in humans. Previous studies were short-term and applied on rats and in normoxic conditions. Similarly, Wozniak et al. evaluated the influence of exercise in high-altitude conditions (about $2000 \mathrm{~m}$ a.s.1.) on SOD and CAT activities in 10 kayakers and 10 rowers. They found a significant increase of SOD and CAT activities in erythrocytes after the $4^{\text {th }}, 10^{\text {th }}$ and $18^{\text {th }}$ day of training [44]. It is known that continuous and intermittent efforts under hypoxic conditions increase oxidative stress $[23,24,45]$. Bailey et al. demonstrated that $60 \mathrm{~min}$ of simulated training under hypoxic conditions significantly increased the levels of serum lipid peroxides, with a simultaneous reduction of antioxidant enzyme activities [46]. Gonzalez et al. [47] and Pialoux et al. [48] reported diminished MDA levels in the plasma of swimmers after an acute hypoxic swimming test (10 $\min$ at $4,800 \mathrm{~m}$ ) as well as in cyclists who spent 13 days at an altitude of 2, 500 to 3,000 m, and trained at $1200 \mathrm{~m}$ above sea level.

SOD is one of the main antioxidant enzymes that degrade superoxide radicals [46]. Increase in SOD enzyme activity corresponds with enhanced resistance to oxidative stress. Groussard et al. found that SOD activity decreased after a single sprint anaerobic exercise [40]. Not all studies reported decrease in SOD in response to exercise. It has been reported that 8-week moderate intensity of aerobic training did not elevate SOD activity. Furthermore, it has been revealed a decrease in SOD levels an acute bout of exercise in skiers participating in a graded treadmill test to exhaustion and elevated erythrocyte SOD activity immediately post exercise when the sprinters performed a sprint exercise [49].

CAT activity in response to exercise is variable. Following about of submaximal exercise a decrease in erythrocyte CAT activity reported in trained cyclists. Furthermore, it has been reported that sprinters who performed a sprint type exercise did not have altered erythrocyte CAT activity [49].

GPX activity is a key component of the glutathione homeostasis and its response to exercise is variable [35]. Higher in oxygen consumption during exercise activates the enzyme GPX to remove hydrogen peroxide. In response to an acute bout of HIIT, elevated erythrocyte GPX activity has been found after a sprint exercise but no change when runners performed an endurance exercise [49].

\section{Conclusion.}

The present study is the first to report improvements in oxidative status after 4 and 8 week high intensity interval training. We speculate that changes in these parameters might represent an increase in ROS after high intensity interval training.

In comparison with other investigators, we believe the present study provided the first direct analysis of effect of high intensity interval training on moderately trained males' antioxidant status hypoxic conditions in a normobaric environment after training.

The results of this study also suggest that interaction effect (timexgroup) for SOD, CAT, GPX and MDA revealed no significant in repeated response. However, it was observed that 8 weeks of high-intensity interval training affected significantly only SOD and GPX. The normoxic and hypoxic conditions were not present between treatments. The limitation of our study was no standardization of dietary habits of the participants. It can be advised to monitor dietary habits of the participants and to apply the same applications to elite athletes for the future studies.

\section{Funding}

This study was supported by University of Ankara, Turkey.

\section{Conflict of interest}

There were no conflicts of interest. 


\section{References}

1. Bloomer RJ, Fisher-Wellman KH. Blood oxidative stress biomarkers: influence of sex, exercise training status, and dietary intake. Gender Medicine, 2008; 5(3): 218-228. https://doi.org/10.1016/j.genm.2008.07.002

2. Gomez-Cabrera MC, Martinez A, Santangelo G, Pallard'o FV, Sastre J, Vina J. Oxidative stress in marathon runners: interest of antioxidant supplementation. The British Journal of Nutrition, 2006; 96(1): 31-33. https://doi.org/10.1079/BJN20061696

3. Halliwell B. Reactive oxygen species in living systems: source, biochemistry, and role in human disease. American Journal of Medicine, 1991; 91(3): 14-22. https://doi.org/10.1016/0002-9343(91)90279-7

4. Berzosa C, Cebrián I, Fuentes-Broto L, Gómez-Trullén E, Piedrafita E, Martínez-Ballarín E, et al. Acute Exercise Increases Plasma Total Antioxidant Status and Antioxidant Enzyme Activities in Untrained Men. Journal of Biomedicine and Biotechnology, 2011;2011:1-7. https://doi. org/10.1155/2011/540458

5. Forsberg L, de Faire U, Morgenstern R. Oxidative stress, human genetic variation, and disease. Arch Biochem Biophys, 2001; 389(1): 84-93. https://doi.org/10.1006/abbi.2001.2295

6. Cooke M, Evans M, Dizdaroglu M, Lunec J. Oxidative DNA damage: mechanisms, mutation, and disease. FASEB J, 2003;17(10):1195-1214. https://doi.org/10.1096/fj.02-0752rev

7. Banarje AK, Mandal A, Chanda D, Chakraborti S. Oxidant, antioxidant and physical exercise. Mol Cell Biochem, 2003; 253(1-2): 307-312. https://doi.org/10.1023/A:1026032404105

8. Koska J, Blazicek P, Marko M, Grna JD, Kvetnansky R, Oigas M. Insulin, catecholamines, glucose and antioxidant enzymes in oxidative damage during different loads in healthy humans. Physiol Res, 2000; 49(19): 95-100.

9. Ji LL. Free radicals and exercise: implication in health and fitness. J Exerc Sci Fitness, 2003; 1(1): 15-22.

10.Kanter M. Free radicals, exercise and antioxidant supplementation. Proc Nutr Soc, 1988; 57(1): 9-13. https://doi.org/10.1079/PNS19980004

11.Powers SK, Criswell D, Lawler J, Martin D, Lieu FK, Ji LL, Herb RA. Rigorous exercise training increases superoxide dismutase activity in ventricular myocardium. Am J Physial, 1993; 6(2) 2094-2098. https://doi.org/10.1152/ajpheart.1993.265.6.H2094

12.Brugniaux JV, Rock E, Schmitt L, Richalet JP. Antioxidant status of elite athletes remains impaired 2 weeks after a simulated altitude training camp. European Journal of Nutrition, 49(5); 285-292. https://doi.org/10.1007/s00394-009-0085-z

13.Barker AR, Day J, Smith A, Bond B, Williams CA. The influence of 2 weeks of low-volume high intensity interval training on healtj outcomes in adolescent boys. Journal of Sport Sciences, 2014; 32:(8):757-765. https://doi.org/10.1080/02640414.2013.853132

14.Shing CM, Webb JJ, Driller MW, Williams AD, Fell JW. Circulating adiponectin concentration and body composition arealteredinresponsetohighintensityintervaltraining.Jornalof Strength and Conditioning Research, 2013;27(8): 2213-2218. https://doi.org/10.1519/JSC.0b013e31827e1644

15.Greeley SJ, Martinez N, Campbell BI. The impact of high-intensity interval training on metabolic syndrome. Strength and Conditioning Journal, 2013;35(2): 63-65. https://doi.org/10.1519/SSC.0b013e31827764da
16.GibalaMJ,LittleJP,MacDonaldMJ,HawleyJA.Physiological adaptations to low-volume, high-intensity interval training in health ve disease. $J$ Physiol, 2012; 590(5):1077-1084. https://doi.org/10.1113/jphysiol.2011.224725

17.Metin G, Gumustas MK, Uslu E, Belce A, Kayserilioglu A. Effects of regular training on plasma thiols, malondialdehyde and carnitine concentrations in young soccer players. Chin J Physiol, 2003;46(1):35-39.

18. Bloomer RJ, Smith WA. Oxidative stress in response to aerobic and anaerobic power testing: Influence of exercise training and carnitine supplementation. Res Sports Med, 2009;17(1):1-16. https://doi.org/10.1080/15438620802678289

19.Shi M, Wang X, Yamanaka T, Ogita F, Nakatani $\mathrm{K}$, Takeuchi T. Effects of anaerobic exercise and aerobic exercise on biomarkers of oxidative stress. Environ Health Prev Med, 2007;12(5):202-208. https://doi.org/10.1265/ehpm.12.202

20.Fisher-Wellman K, Bloomer RJ. Acute exercise and oxidative stress: a 30-year history. Dyn Med, 2009;8(1):1-25. https://doi.org/10.1186/1476-5918-8-1

21.Guoxiang Y, Gautam A, Andrew A. McCormick JJH, Ganesh KK, Nanduri RP. Role of oxidative stress in intermittent hypoxia-induced immediate early gene activation in rat PC12 cells. $J$ Physiol, 2004; 557(33):773-783. https://doi.org/10. 1113/jphysiol. 2003. 058503

22.Askew EW. Work at high altitude and oxidative stress: antioxidant nutrients. Toxicology, 2002;180(2):107-119. https://doi.org/10.1016/S0300-483X(02)00385-2

23.Bailey DM, Davies B, Young IS. Evidence for reactive oxidant generation during acute physical exercise and normobaric hypoxia in men. J Physiol, 2000; 528-599.

24.Bailey DM, Davies B, Young IS. Intermittent hypoxia training implications for lipid peroxidation induced by acute normoxic exercise in active men. Clin Sci Lond, 2001;101(5): 465-475. https://doi.org/10.1042/cs1010465

25.Moller P, Loft S, Lundby C, Olsen NV. Acute hypoxia and hypoxic exercise induce DNA strand breaks and oxidative DNA damage in humans. FASEB $J, 2001 ; 15(7): 1181-1186$. https://doi.org/10.1096/fj.00-0703 com

26.Furman YM, Holovkina V, Salnykova S, Sulyma A, Brezdeniuk O, Korolchuk A, Nesterova S. Effect of swimming with the use of aqua fitness elements and interval hypoxic training on the physical fitness of boys aged 1112 years. Pedagogics, psychology, medical-biological problems of physical training and sports. 2018;22(4):184-8. https://doi.org/10.15561/18189172.2018.0403

27.Rodriguez FA, Murio J, Ventura JL. Effects of intermittent hypobaric hypoxia and altitude training on physiological and performance parameters in swimmers. Med Sci Sports Exerc, 2003;35(5):115. https://doi.org/10.1097/00005768-200305001-00624

28.Rodriguez FA, Truijens MJ, Townsend NE, Stray-Gundersen J, Gore CJ, Levine BD. Performance of runners and swimmers after four weeks of intermittent hypobaric hypoxic exposure plus sea level training.JApplPhysiol,2007;103(5):1523-1535. https://doi.org/10.1152/japplphysiol.01320.2006

29.Czuba M, Maszczyk A, Gerasimuk D, Roczniok R, FidosCzuba O, Zając A, et al. The effects of hypobaric hypoxia on erythropoiesis, maximal oxygen uptake and energy cost of exercise under normoxia in elite biathletes. JSSM, 2014;13(4):912-920.

30.Czuba M, Waskiewicz Z, Zajac A, Poprzecki S, Cholewa J, Roczniok R. The effects of intermittent hypoxic training on aerobic capacity and endurance performance in cyclists. $J$ Sports Sci Med, 2011;10(1):175-183. 
31.Poprzecki S, Czuba M, Zając A, Karpinski J, Wilk R, Bril G, Maszczyk A, Toborek M. The blood antioxidant defence capacity during intermittent hypoxic training in elite swimmers. Biol. Sport, 2016: 33(4):353-360. https://doi.org/10.5604/20831862.1221607

32.Dahle LK, Hill EG, Holman RT. The thiobarbituric acid reaction and the autoxidations of polyunsaturated fatty acid methyl esters. Arch Biochem Biophys, 1962; 98(2): 253-261. https://doi.org/10.1016/0003-9861(62)90181-9

33.Durak İ, Canbolat O, Kavutcu M, Öztürk HS, Yurtarslanı Z. Activities of total, cytoplasmic and mitochondrial superoxide dismutase en $\neg$ zymes in sera and pleural fluids from patients with lung cancer. J Clin Lab Anal, 1996; 10(1): 17--20. h t t p s://doi org/10.1002/( S I C I ) 1098 2825(1996)10:1<17::AID-JCLA4>3.3.CO;2-6

34.Paglia DE, Valentine WN. Studies on the quantitative and qualitative characterization of erythrocyte glutathione peroxidase. J Lab Clin Med, 1967; 70(1): 158-169.

35.Aebi H. Catalase. In: Bergmayer HU, ed. Methods of Enzymatic Analysis. New York and London: Academic Press Inc, 1974: 673-677. https://doi.org/10.1016/B978-0-12-091302-2.50032-3

36. Wadley AJ, Chen Y, Lip GYH, Fisher JP, Aldred S. Low volume high intensity interval exercise elicits antioxidant and anti-inflammatory effects in humans. Journal of Sports Science, 2016;34(1);1-9. https://doi.org/10.1080/02640414.2015.1035666

37. Criswell D, Powers S, Dodd S, Lawler J, Edwards W, Renshler K, Grinton S. High intensity training induced changes in skeletal muscle antioxidant enzyme activity. Med. Sci. Sports and Exercise, 1993; 25(10):1135-1140. https://doi.org/10.1249/00005768-199310000-00009

38.Ugras AF. Effect of high intensity interval training on elite athletes' antioxidant status. Sci Sports, 2013;28(5):253-259. https://doi.org/10.1016/j.scispo.2012.04.009

39.Packer L, Cadenas E, Davies KJA. Free radicals and exercise: an introduction. Free Radic Biol Med, 2008; 44(2):123-125. https://doi.org/10.1016/j.freeradbiomed.2007.05.031

40.Groussard C, Rannou-Bekono F, Machefer G, Chevanne M, Vincent S, Sergent O, et al. Changes in blood lipid peroxidation markers and antioxidants after a single sprint anaerobic exercise. Eur J Appl Physiol, 2003;89(1):14-20. https://doi.org/10.1007/s00421-002-0767-1

41.Shi $M$, Wang $X$, Yamanaka $T$, Ogita $F$, Nakatani $\mathrm{K}$, Takeuchi T. Effects of anaerobic exercise and aerobic exercise on biomarkers of oxidative stress. Environ Health Prev Med, 2007;12(5):202-208. https://doi.org/10.1265/ehpm.12.202

42.Fisher-Wellman K, Bloomer RJ. Acute exercise and oxidative stress: a 30-year history. Dyn Med, 2009;8(1):1-25. https://doi.org/10.1186/1476-5918-8-1

43.Kinnunen S, Atalay M, Hyypa S, Lehmuskero A, Hanninen O, Oksala N. Effects of prolonged exercise on oxidative stress and antioxidant defense in endurance horse. $J$ Sports Sci Med, 2000; 4(4):415-421.

44. Wozniak A, Drewa G, Chesy G, Rakowski A, Rozwodowska M, Olszewska D. Effect of altitude training on the peroxidation and antioxidants enzymes in sportsmen. Med Sci Sports Exerc, 2001;33(7):1109-1113. https://doi.org/10.1097/00005768-200107000-00007

45.Risby TH, Sehnert SS. Clinical application of breath biomarkers of oxidative stress status. Free Radic Biol Med, 1999;27(11-12):1182-1192. https://doi.org/10.1016/S0891-5849(99)00212-9

46. Qiao D, Hou L, Liu X. Influence of intermittent anaerobic exercise on Mouse physical endurance and antioxidant components. $\mathrm{Br} \quad J$ Sports Med, 2006;40(3):214-218. https://doi.org/10.1136/bjsm.2005.020099

47.Gonzalez G, Celedon G, Escobar M, Sotomayor C, Ferrer V, BenitezD, BehnC. Red cell membrane lipid changes at 3,500 m andonreturntosealevel.HighAltMedBiol,2005;6(4):320-326. https://doi.org/10.1089/ham.2005.6.320

48.Pialoux V, Mounier R, Ponsot E, Rock E, Mazur A, Dufour S, Richard R, Richalet JP, Coudert J, Fellmann N. Effects of exercise and training in hypoxia on antioxidant/prooxidant balance. Eur J Clin Nutr, 2006;60(12):1345-1354. https://doi.org/10.1038/sj.ejen.1602462

49.Urso ML, Clarkson PM. Oxidative stress, exercise and antioxidant supplementation. Toxicology, 2003;189(1-2):41-45. https://doi.org/10.1016/S0300-483X(03)00151-3

\section{Information about the authors:}

Akgul M.S.; (Corresponding author); https://orcid.org/0000-0002-9696-6541; msakirakgul@gmail.com; School of Physical Education and Sports, Kastamonu University; 37150 Kastamonu, Turkey.

Koz M.; https://orcid.org/0000-0002-5793-6999; mkoz@ankara.edu.tr; Faculty of Sport Sciences, Ankara University; 06830 Ankara, Turkey.

\section{Cite this article as:}

Akgul MS, Koz M. Effect of high intensity interval training under hypoxic conditions in a normobaric environment on moderately trained university students' antioxidant status. . Physical education of students, 2019;23(5):217-222. https://doi.org/10.15561/20755279.2019.0501

This is an Open Access article distributed under the terms of the Creative Commons Attribution License, which permits unrestricted use, distribution, and reproduction in any medium, provided the original work is properly cited http://creativecommons.org/licenses/by/4.0/deed.en

Received: 07.08.2019

Accepted: 03.09.2019; Published: 26.10 .2019 